\title{
In vitro Eye Irritancy Test of Lauryl Derivatives and Polyoxyethylene Alkyl Derivatives with the Reconstructed Rabbit Corneal Epithelium
} Model

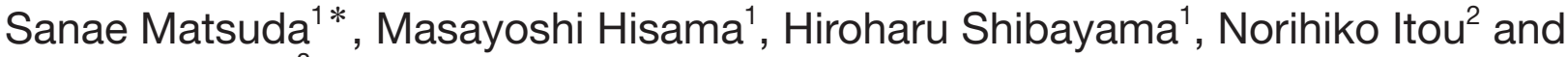 \\ Masahiro Iwaki ${ }^{3}$ \\ ${ }^{1}$ HBC Science Research Center Co., Ltd (3-13-9, Higashinakamoto, Higashinari-ku, Osaka, 537-0021, JAPAN) \\ ${ }^{2}$ Department of Ophthalmology, Yokohama City University School of Medicine (3-9, Fukuura, Kanazawa-ku, Yokohama, Kanagawa 236- \\ 0004, JAPAN) \\ ${ }^{3}$ Department of Pharmacy, School of Pharmacy, Kinki University (3-4-1, Kowakae, Higashi-Osaka, Osaka 577-8502, JAPAN)
}

\begin{abstract}
The rabbit corneal epithelium model (RCE model) was developed as a three-dimensional in vitro model to replace animal testing for the assessment of eye tolerance. In the model, a stratified culture of rabbit corneal epithelial cells is grown at the air-liquid interface on a collagen gel acting as a parabasal membrane. Histological cross-sections show that the structure of RCE model closely parallels that of the rabbit corneal epithelium. The lauryl derivatives, such as sodium lauryl sulfate (SLS), polyoxyethylene (9) lauryl ether (PLE), sodium polyoxyethylene (2) lauryl ether sulfate (SPLE), mono glyceryl laurate (MGL), and sodium N-lauroyl-L-glutaminate (SLG), and polyoxyethylene alkyl derivatives, polyoxyethylene (9) lauryl ether (PLE), polyoxyethylene (10) cetyl ether (PCE), polyoxyethylene (10) stearyl ether (PSE), polyoxyethylene (10) oleyl ether (POE), and polyoxyethylene (10) behenyl ether (PBE), were evaluated for in vitro eye irritation potential using the RCE model by the measurement of viability with MTT assay. SLS, PLE, SPLE, MGL, and SLG inhibited $90.3 \%, 69.8 \%, 79.7 \%, 45.8 \%$, and $32.7 \%$ of the viability at a concentration of $0.5 \%$. The IC50 (50\% inhibitory concentration) values of SLS, PLE, SPLE, MGL, and SLG were $0.086 \%, 0.205 \%, 0.133 \%, 0.627 \%$, and $0.934 \%$, respectively. These results indicated that a functional group at the end of lauryl chain is an important factor for inhibiting the viability using the RCE model. The polyoxyethylene alkyl derivatives had distinctly different the viability potencies according to their alkyl patterns. PLE inhibited the viability greater than other polyoxyethylene alkyl derivatives. Therefore, the lauryl chain of PLE is an important factor for inhibiting the viability on the RCE model.
\end{abstract}

Key words: eye irritation, rabbit corneal epithelium model, rabbit corneal epithelial cell, cosmetic integrants, MTT

\section{INTRODUCTION}

The in vivoDraize eye test ${ }^{1}$, has become the international standard assay for acute eye irritation (OECD TG 405) and continued to be the method of choice for the regulatory toxicology safety assessment of eye irritation hazard of cosmetic products and ingredients in many countries since 1940s. However, the increase in animal use has drawn the public attention to issues such as animal welfare and 3Rs (reduction of the number of animals used, refinement of techniques and procedures to reduce pain and distress, replacement of animal techniques with non-animal techniques $)^{2)}$ in recent years.

The $7^{\text {th }}$ amendment of the Cosmetic directive will lead to the ban of animal testing for cosmetic ingredients in 2009. Thus, alternative strategies are necessary which allow the testing of chemicals with wide physicochemical properties under conditions similar to in vivo exposure. Many efforts have been made in order to find reliable and relevant predictive models such as the chorioallantoic membrane (CAM) methods ${ }^{3)}$, cell-based cytotoxicity methods ${ }^{4,5)}$, recon-

*Correspondence to: Sanae Matsuda, HBC Science Research Center Co., Ltd, 3-13-9, Higashinakamoto, Higashinari-ku, Osaka, 5370021, JAPAN

E-mail: matsuda@hbc-science.co.jp

Accepted April 21, 2009 (received for review March 16, 2009)

Journal of Oleo Science ISSN 1345-8957 print / ISSN 1347-3352 online

http://www.jstage.jst.go.jp/browse/jos/ 


\section{S. Matsuda, M. Hisama, H. Shibayama et al.}

stituted tissue models ${ }^{6,7)}$, and isolated organ methods ${ }^{8}$. Success in fully replacing the Draize eye irritation test with in vitro methods has not yet occurred, in part due to a lack of understanding of the underlying physiological mechanisms of eye irritation.

We developed the Rabbit Corneal Epithelial (RCE) model using cultured rabbit corneal epithelial cells and collagen gel as a scaffold to evaluate in vitro the eye irritation potential of chemicals including pharmaceuticals, cosmetics and their raw ingredients. The collagen gel has good physical properties of strength and elasticity, and so is a useful scaffold for the RCE model. Generally, cytotoxicity tests using cultured cells have the advantage of being simple and quick with, a low evaluation cost. However, when using cultured cells alone in media, the testing of water insoluble materials is sometimes difficult as the test substances may precipitate out in the media. In contrast to the conventional monolayer culture system suspended in media, the RCE model has a dry surface. Therefore, it is useful for both soluble and insoluble substances including various forms of cosmetic products. The aim of the development of RCE model was to evaluate a new three-dimensional epithelial model cultivated from rabbit corneal cells to replace animal testing in the assessment of eye tolerance. Thus we focused on the mechanisms of eye injury, and examined new in vitro endpoints which would be more predictive of the biogenic response to chemical injury. This should help to develop new or improved in vitro methods that could proceed to formal validation.

Lauryl fatty chain derivatives are one group of surfactants which includes anionic, cationic and nonionic surfactants with various functional groups. Surfactants containing lauryl fatty chain are widely used for toiletry products and cosmetics. Of those, the lauryl derivatives such as polyoxyethylene lauryl ether, sodium polyoxyethylene lauryl ether sulfate, mono glyceryl laurate, and sodium N-lauroyl-L-glutaminate are frequently used in shampoo as a foaming agent and in skin-care cosmetics as an emulsifier. Therefore, it is important to evaluate the eye safety of lauryl fatty chain derivatives and to elucidate the mechanism of action of eye irritation.

Polyoxyethylene alkyl derivatives, such as polyoxyethylene lauryl ether, polyoxyethylene cetyl ether, polyoxyethylene stearyl ether, polyoxyethylene oleyl ether, and polyoxyethylene behenyl ether, are one group of well-known nonionic surfactants, and have been widely used in toiletry products and cosmetics. In Japan, there have been reported occurrences of dangerous damage to eyes through the use of make-up removers combined with polyoxyethylene oleyl ethers. The Japan Cosmetic Industry Association (JCIA) has warned that use of make-up removers combined with polyoxyethylene oleyl ethers could result in penumbra of the eye. Therefore, it is important to evaluate whether polyoxyethylene oleyl ethers are safe for eye treatment, and to elucidate the mechanism of action of the eye irritation.

In this study, we evaluated the RCE model, which is cultured using cultured rabbit corneal epithelial cells and collagen gel as a scaffold, to evaluate in vitro eye irritation potential of lauryl derivatives and polyoxyethylene alkyl derivatives, such as surfactants. Moreover, we investigated the activity-structure relationship of surfactants, such as lauryl derivatives and polyoxyethylene alkyl derivatives on in vitro eye irritation potential.

\section{EXPERIMENTAL}

\subsection{Materials}

Sodium lauryl sulfate (SLS) and MTT (3-(4,5-dimethylthiazol)-2,5-diphenyl-tatrazolium bromide) were obtained from Wako Pure Chemical Industries, Co. Ltd. (Tokyo, Japan). Polyoxyethylene (9) lauryl ether (PLE), sodium polyoxyethylene (2) lauryl ether sulfate (SPLE), mono glyceryl laurate (MGL), sodium N-lauroyl-L-glutaminate (SLG), polyoxyethylene (10) cetyl ether (PCE), polyoxyethylene (10) stearyl ether (PSE), polyoxyethylene (10) oleyl ether (POE), and polyoxyethylene (10) behenyl ether (PBE), which were selected from the Japanese Standard of Cosmetic Ingredients or Japanese Pharmacopoeia, were obtained from a supplier of cosmetic ingredients.

\subsection{Cell and culture}

Normal rabbit corneal epithelial cells (NRCE) were provided by ArBlast Co. Ltd. (Kobe, Japan) and were co-cultured along with inactivated 3T3 fibroblasts, as described previously ${ }^{9}$. The culture medium M-stars A and supplemented hormonal epithelial medium (SHEM) were obtained from ArBlast Co. Ltd. (Kobe, Japan). For three-dimensional cell culture, type-I collagen was prepared on ice according to the manufacturer's instructions (Nitta Gelatin Inc., Japan). The type-I collagen mixture was added to polyester membrane culture inserts and allowed to gel at $37^{\circ} \mathrm{C}$. After that, the culture inserts were placed in a 12-well plate containing the treated 3T3 fibroblasts, and the cultured rabbit corneal epithelial cells were inoculated on 12-well culture inserts. The culture was submerged into the previously described M-stars A medium for 5 days, and then exposed to air by lowering the medium level (airlifting). After airlifting, the suitability of the rabbit corneal epithelial (RCE) model was confirmed by examining the multilayer structure formation and the flatness of the most outer layer.

\subsection{Light microscopy}

The samples were fixed with $10 \%$ buffered neutral formaldehyde. Then, the samples were routinely processed by cryostat and were stained with hematoxylin and eosin. 


\subsection{Eye irritation tests with the RCE model}

A test sample (100 $\mu \mathrm{L}$, diluted with PBS) was applied to the surface of the RCE model, and the tissue was cultured in a SHEM medium at $37^{\circ} \mathrm{C}$ under $5 \% \mathrm{CO}_{2}$ for $30 \mathrm{~min}$. After incubation, the eye irritation potency of the test samples was estimated by the measurement of cytotoxicity using the MTT assay ${ }^{10}$. Briefly, tissues were quickly blotted on absorbent paper and transferred to $0.5 \mathrm{mg} / \mathrm{mL}$ MTT in maintenance medium $(1.2 \mathrm{~mL} /$ well of a 12 well plate $)$ and incubated for $3 \mathrm{~h}$. RCE models were removed from the MTT solution and again blotted on absorbent paper and transferred to isopropanol $(1.5 \mathrm{~mL} /$ well and $0.5 \mathrm{~mL}$ added onto each tissue) for $2 \mathrm{~h}$ at room temperature with protection from evaporation and light (formazan extraction). After 15 min of gentle shaking, $200 \mu \mathrm{L}$ aliquots were transferred to a flat bottom 96 well plate before measuring the optical density at $570 \mathrm{~nm}$ with isopropanol as the blank, using a microtiter plate reader. The viability rate (\% of control) is expressed as \% relative to the PBS non-irritation control.

Viability rate $(\%$ of control $)=[(\mathrm{OD}$ with irritant $-\mathrm{OD}$ blank) / (OD without irritant - OD blank)] × 100

\subsection{Statistical analysis}

The inhibitory effect on the cytotoxicity was expressed as the mean \pm standard error (S.E.) of three independent experiments, and subsequent inspection of the means was evaluated using the Student's t-test between two groups at a significance level of $p<0.01$.

\section{RESULTS}

\subsection{Preparation of the rabbit corneal epithelial model}

Normal rabbit corneal epithelial cells (NRCE) have been used to develop a three-dimensional in vitro model of the rabbit corneal epithelium (RCE model). NRCE cells form a stratified culture when grown at the air-liquid interface on collagen gel as a scaffold in a culture insert with serumfree M-stars A medium. The RCE model was confirmed to be a multilayer of well-stratified corneal cells on collagen gel acting as a scaffold by examining a histological vertical section (Fig. 1).

\subsection{Dose effect relationship of the in vitro eye irritation test on the RCE model}

To investigate the in vitro eye irritation potential of integrants, we evaluated the viability of RCE model with integrants. The percent viability of the reference chemical sodium lauryl sulfate (SLS), which is classed as severe and extreme level of eye irritation on the Draize score and show the Draize score in a dose-dependent manner, obtained at seven concentrations were compared using 6 parallel wells at each concentration. Test data indicated

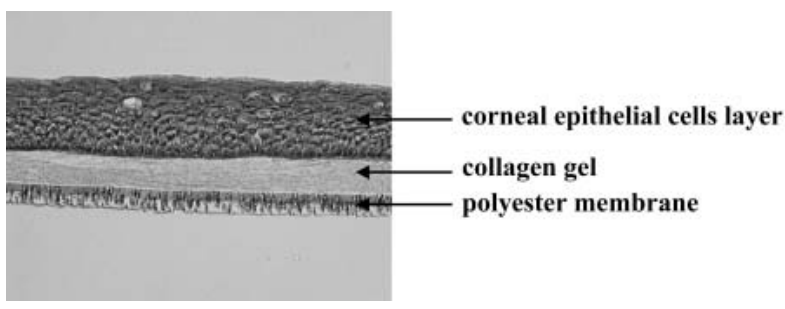

Fig. 1 Microscopic View of a Histological Vertical Section of the Rabbit Corneal Epithelium (RCE) Model $(\times 200)$.

The vertical section was stained with hematoxylin/eosin.

the percent viability calculated from the RCE model decreased in response to an increase in SLS concentration (Table 1). As illustrated in Fig. 2, a dose-effect relationship was observed where lower viability was obtained at higher concentrations of SLS $(F=1055.362$ and $P<0.001$ from a single factor analysis of variance of the results). SLS inhibited $90.3 \%$ of the viability at a concentration of $0.5 \%$ in the $\mathrm{RCE}$ model. The $50 \%$ inhibition concentration $\left(\mathrm{IC}_{50}\right)$ value of SLS was $0.078 \%$ in RCE model.

\subsection{Eye irritation test of lauryl derivatives with the RCE model}

To investigate the eye irritation of lauryl derivatives, we evaluated the viability of the RCE model with treatment of the lauryl derivatives such as polyoxyethylene (9) lauryl ether (PLE), sodium polyoxyethylene (2) lauryl ether sulfate (SPLE), mono glyceryl laurate (MGL), and sodium N-lauroyl-L-glutaminate (SLG). These all lauryl derivatives decreased the viability of the RCE model in a dose-dependent manner (Fig. 3). PLE, SPLE, MGL, and SLG inhibited $69.8 \%, 79.7 \%, 45.8 \%$, and $32.7 \%$ of the viability at a concen-

Table 1 Results from the in vitro Eye Irritation Test using the RCE Model at Different Concentrations of SLS. The cell viability represents the mean \pm S.D. of six experiments compared with the control group.

\begin{tabular}{cccc}
\hline $\begin{array}{c}\text { Concentration } \\
(\%)\end{array}$ & $\begin{array}{c}\text { Viability } \\
\text { (\% of control) }\end{array}$ & $F$ & $P$ \\
\hline 0.0125 & $90.566 \pm 2.222$ & & \\
0.025 & $80.136 \pm 2.3256$ & & \\
0.050 & $63.020 \pm 3.560$ & & \\
0.125 & $34.1332 \pm 2.909$ & 1055.362 & $<0.001$ \\
0.250 & $17.5632 \pm 1.4576$ & & \\
0.500 & $9.6546 \pm 1.1542$ & & \\
1.000 & $4.0402 \pm 0.4189$ & & \\
\hline
\end{tabular}




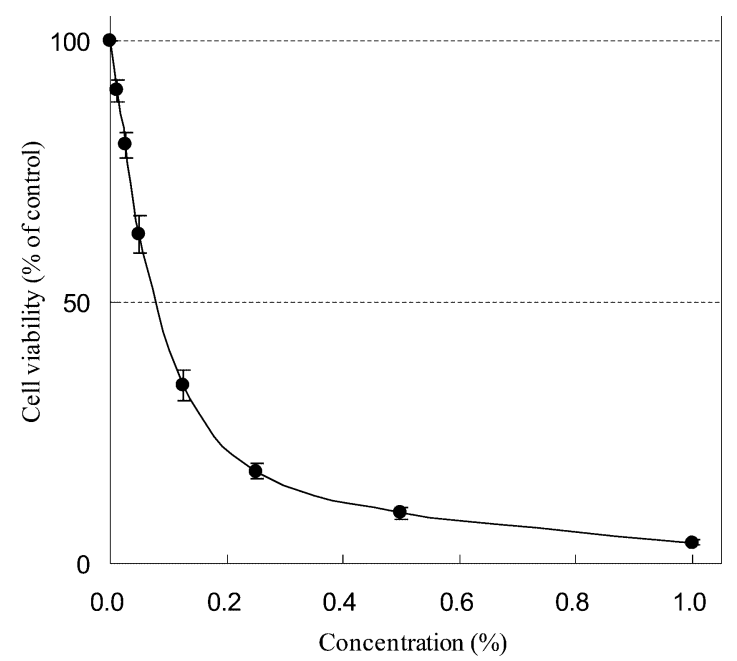

Fig. 2 Cell Cytotoxicity of Sodium Lauryl Sulfate (SLS) in the in vitro Eye Irritation Test Using the RCE Model.

Cell cytotoxicity was measured using the MTT assay. The cell viability represents the mean \pm S.D. of six experiments compared with the control group.

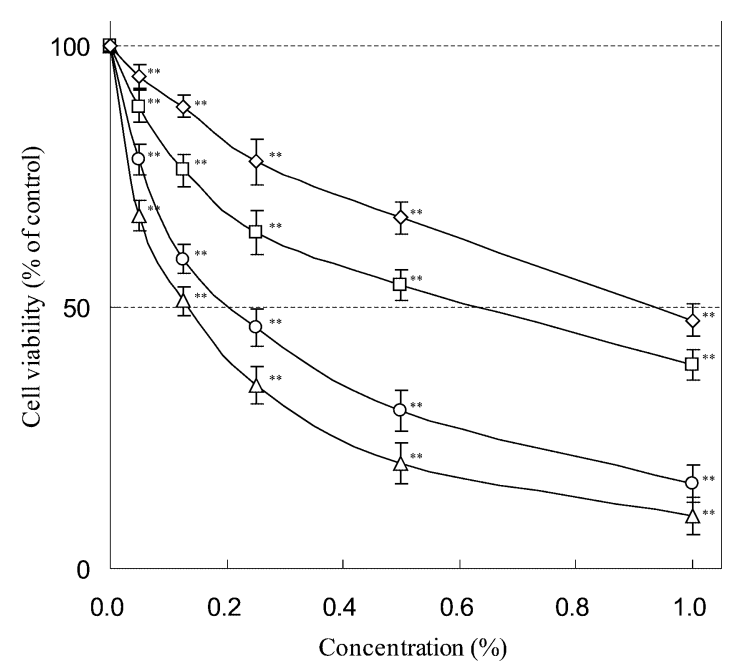

Fig. 3 Cell Cytotoxicity of Lauryl Derivatives from the in vitro Eye Irritation Test Using the RCE Model. ( $(\bigcirc)$ PLE; $(\triangle)$ SPLE; $(\square)$ MGL; $(\diamond)$ SLG).

Cell cytotoxicity was measured using the MTT assay. The cell viability represents the mean \pm S.D. of six experiments compared with the control group. Significantly different from the non-treated control group at $p<0.01(* *)$. tration of $0.5 \%$ in $\mathrm{RCE}$ model, and the $\mathrm{IC}_{50}$ values of PLE, SPLE, MGL, and SLG were $0.205 \%, 0.133 \%, 0.627 \%$, and $0.934 \%$, respectively. SLS inhibited the viability greater than all lauryl derivatives on RCE model.

\subsection{Eye irritation test of polyoxyethylene alkyl derivatives with the RCE model}

To investigate the eye irritation of polyoxyethylene alkyl derivatives, we evaluated the viability of the RCE model with treatment of the polyoxyethylene alkyl derivatives such as polyoxyethylene (9) lauryl ether (PLE), polyoxyethylene (10) cetyl ether (PCE), polyoxyethylene (10) stearyl ether (PSE), polyoxyethylene (10) oleyl ether (POE), and polyoxyethylene (10) behenyl ether (PBE). These all polyoxyethylene alkyl derivatives decreased the viability of the RCE model in a dose-dependent manner (Fig. 4). PLE, PCE, PSE, POE, and PBE inhibited 69.8\%, 52.1\%, 57.8\%, $63.5 \%$, and $38.7 \%$ of the viability at a concentration of $0.5 \%$ in RCE model, and the IC50 value of PLE, PCE, PSE, POE, and $\mathrm{PBE}$ were $0.205 \%, 0.455 \%, 0.357 \%, 0.275 \%$, and $0.765 \%$, respectively. PLE inhibited the viability greater than other polyoxyethylene alkyl derivatives on RCE model.

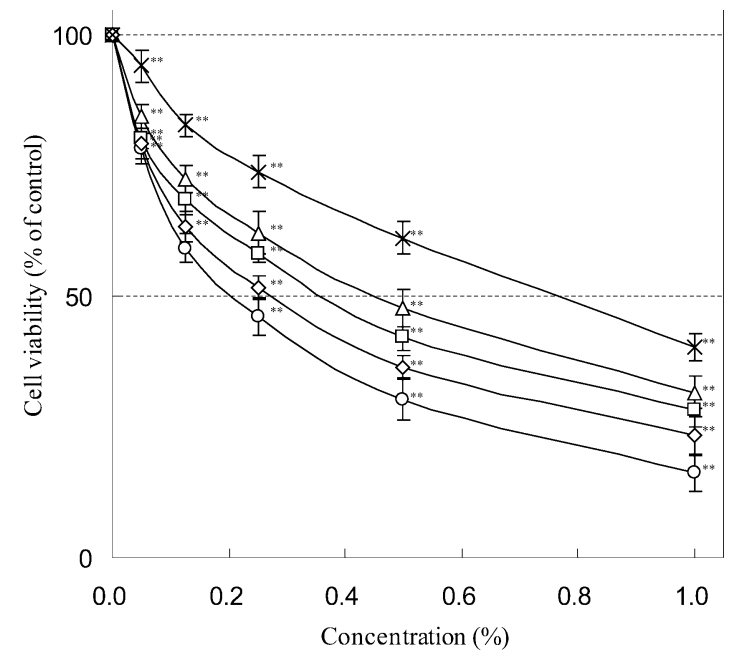

Fig. 4 Cell Cytotoxicity of Polyoxyethylene Alkyl Derivatives from the in vitro Eye Irritation Test Using the RCE Model.

$((\bigcirc)$ PLE; $(\triangle)$ PCE; $(\square)$ PSE; $(\diamond)$ POE; $(\times)$ PBE). Cell cytotoxicity was measured using the MTT assay. The cell viability represents the mean \pm S.D. of six experiments compared with the control group. Significantly different from the nontreated control group at $p<0.01(* *)$. 


\section{In vitro Eye Irritancy Test of Lauryl Derivatives and Polyoxyethylene Alkyl Derivatives}

\section{DISCUSSION}

We developed the rabbit corneal epithelium (RCE) model as a three-dimensional in vitro model using a stratified culture of rabbit corneal epithelial cells grown at the air-liquid interface on collagen gel as a parabasal membrane. This model was confirmed to be a multilayer of well-stratified corneal cells (Fig. 1). This allows an artificial corneal epithelium (reconstituted rabbit corneal epithelium) to be prepared which exhibits barrier characteristics and paracellular permeability similar to those of native rabbit cornea.

Eye irritation caused by exogenous chemical irritants usually involves damage to the cornea, conjunctive epithelium and endothelial cells. SLS, which are classed as severe and extreme level of eye irritation on the Draize scores, decreased the viability of the RCE models in a dose-dependent manner (Fig. 2). These results were comparable with those of the Draze test ${ }^{11,12}$, and therefore, the degree of eye irritation caused by chemicals can be simulated using the RCE model as an in vitro eye irritation test.

As for the structure-activity relationships, the lauryl derivatives had distinctly different inhibitory potencies against the viability in the RCE model, according to their substitution patterns. The inhibitory effects of the viability decreased in the order $\mathrm{SLS}>\mathrm{SPLE}>\mathrm{PLE}>\mathrm{MGL}>\mathrm{SLG}$ (Fig. 3). The difference in structure between the lauryl derivatives, SLS, SPLE, PLE, MGL, and SLG is a functional group at the end of lauryl fatty chain. These results indicated that the functional group at the end of lauryl fatty chain is an important factor for inhibiting the viability in the order sodium sulfate $>$ sodium polyoxyethylene ether sulfate $>$ polyoxyethylene ether $>$ glycerate $>$ sodium glutaminate.

As for the structure-activity relationships, the polyoxyethylene alkyl derivatives had distinctly different inhibitory potencies against the viability according to their alkyl patterns. The inhibitory effects of viability decreased in the order PLE $>$ PSE $>$ PCE $>$ PBE in the polyoxyethylene saturated fatty acid derivative group (Fig. 4). The difference in structure between PLE, PSE, PCE and PBE is the length of the fatty chain. These results indicated that the length of the fatty chain is an important factor for inhibiting the viability on polyoxyethylene saturated alkyl derivatives. Moreover, the unsaturated polyoxyethylene alkyl derivative, $\mathrm{POE}$, inhibited the viability greater than PSE. The difference in structure between POE and the saturated polyoxyethylene alkyl derivative, PSE is the existence of an unsaturated olefinic double bond. These results indicated that the unsaturated olefinic double bond is also an important factor in determining the extent of the viability on the RCE model.

In conclusion, the rabbit corneal epithelial ( $\mathrm{RCE}$ ) model was prepared by culture of rabbit corneal cells on collagen gel in a cell culture insert. The eye irritancy effects of lau- ryl derivatives and polyoxyethylene alkyl derivatives were determined by measurement of the cell viability using the $\mathrm{RCE}$ model as a guideline. The results of this test were comparable with those of the Draize test, and thus this modification of the RCE model may provide a useful and sensitive in vitro eye irritation test to replace the animalbased eye irritation test. In addition, the eye irritation test on the RCE model could remarkably detect the small difference of inhibitory effects in lauryl and polyoxyethylene alkyl derivatives, which could not be confirmed with the Draize test. Thus, the RCE model may be useful for a sensitive in vitro eye irritation test. However, the in vitro eye irritation test using RCE model may not exhibit its expected effects in vivo if it is adversely affected by factors such as absorption, bio-disposition, and metabolism after its incorporation into the human cornea. Further studies with mammalian human cells in vitro or in vivo are needed to determine the irritation of chemicals on human cornea.

\section{References}

1. Draize, J.H. Appraisal of the safety of chemicals in foods, drugs and cosmetics. The Association of Food and Drug Officials of the United States. Austin TX (1959).

2. Balls, M.; Goldberg, A.M.; Fentem, J.H.; Broadhead, C.L.; Burch, R.L.; Festing, M.F.; Frazier, J.M.; Hendriksen, C.F.; Jennings, M.; van der Kamp, M.D.; Morton, D.B.; Rowan, A.N.; Russell, C.; Russell, W.M.; Spielmann, H.; Stephens, M.L.; Stokes, W.S.; Straughan, D.W.; Yager, J.D.; Zurlo, J.; van Zutphen, B.F. The three Rs: The way forward: the report and recommendations of ECVAM Workshop 11. Altern. Lab. Anim. 23, 838866 (1995).

3. Hagino, S.; Kinoshita, S.; Tani, N.; Nakamura, T.; Ono, N.; Konishi, K.; Iimura, H.; Kojima, H.; Ohno, Y. Interlaboratory validation of in vitro eye irritation tests for cosmetic ingredients. (2) Chorioallantoic membrane (CAM) test. Toxicol. in vitro. 13, 99-113 (1999).

4. Uchiyama, T.; Akiyama, J.; Miyai, E.; Sakamoto, K.; Takino, Y.; Ohnuma, M.; Ohkosi, K.; Okamoto, Y.; Morito, Y.; Kojima, H.; Okumura, H.; Sawamura, J.; Ikeda, N.; Sumida, Y.; Chiba, K.; Makino, I.; Kawakami, K.; Yamamoto, R.; Torishima, H.; Yanase, H.; Miyajima, A.; Sunouchi, M.; Hayashi, M.; Ohno, Y. Interlaboratory validation of the in vitro eye irritation tests for cosmetic ingredients. (7) Evaluation of cytotoxicity test by CornePack $^{\circledR}$. Toxicol. in vitro 13, 163-173 (1999).

5. Tani, N.; Kinoshita, S.; Okamoto, Y.; Kotani, M.; Itagaki, H.; Murakami, N.; Sugiura, S.; Usami, M.; Kato, K.; Kojima, H.; Ohno, T.; Saijo, K.; Kato, M.; Hayashi, M.; Ohno, Y. Interlaboratory validation of the in vitro eye irritation tests for cosmetic ingredients. (8) Evaluation 
of cytotoxicity tests on SIRC cells. Toxicol. in vitro 13, 175-187 (1999).

6. Ohuchi, J.; Kasai, Y.; Sakamoto, K.; Ohnuma, M.; Kitamura, M.; Kawasaki, Y.; Kakishima, H.; Suzuki, K.; Kuwahara, H.; Imanishi, Y.; Tatsumi, H.; Kotani, M.; Inoue, K.; Okumura, H.; Arashima, M.; Kurishita, A.; Kinoshita, S.; Tani, N.; Kojima, H.; Nakamura, T.; Suzuki, K.; Ishibashi, T.; Hori, H.; Takahashi, H.; Nishikawa, T.; Kitano, Y.; Ohno, Y. Interlaboratory Validation of the in vitro Eye Irritation tests for cosmetic ingredients. (6) Evaluation of MATREX ${ }^{\mathrm{TM}}$. Toxicol. in vitro. 13, 153162 (1999).

7. Jones, P.A.; Budynsky, E.; Cooper, K.J.; Decker, D.; Griffiths, H.A.; Fentem, J.H. Comparative evaluation of five in vitro tests for assessing the eye irritation potential of hair-care products. Altern. Lab. Anim. 29, 669-692 (2001).

8. Xu, K.P.; Li, X.F.; Yu, F.S. Corneal organ culture model for assessing epithelial responses to surfactants. Toxicol. Sci. 58, 306-314 (2000).

9. Rheinwald, J.G.; Green, H. Serial cultivation of strains of human epidermal keratinocytes: The formation of keratinizing colonies from single cells. Cell. 6, 331-343
(1975).

10. Mosmann, T. Rapid colorimetric assay for cellular growth and survival: Application to proliferation and cytotoxicity assays. J. Immunol. Methods. 65, 55-63 (1983).

11. Ohno, Y.; Kaneko, T.; Inoue, T.; Morikawa, Y.; Yoshida, T.; Fujii, A.; Masuda, M.; Ohno, T.; Hayashi, M.; Momma, J.; Uchiyama, T.; Chiba, K.; Ikeda, N.; Imanishi, Y.; Itagaki, H.; Kakishima, H.; Kasai, Y.; Kurishita, A.; Kojima, H.; Matsukawa, K.; Nakamura, T.; Ohkoshi, K.; Okumura, H.; Saijo, K.; Sakamoto, K.; Suzuki, T.; Takano, K.; Tatsumi, H.; Tani, N.; Usami, M.; Watanabe, R. Interlaboratory validation of the in vitro eye irritation tests for cosmetic ingredients. (1) Overview of the validation study and Draize scores for the evaluation of the tests. Toxicol. in vitro 13, 73-98 (1999).

12. Van Goethem, F.; Adriaens, E.; Alépée, N.; Straube, F.; De Wever, B.; Cappadoro, M.; Catoire, S.; Hansen, E.; Wolf, A.; Vanparys, P. Prevalidation of a new in vitro reconstituted human cornea model to assess the eye irritating potential of chemicals. Toxicol. in vitro $20,1-$ 17 (2006). 